\title{
Spatiotemporal Structure of Olfactory Inputs to the Mushroom Bodies
}

\author{
Gilles Laurent, ${ }^{1}$ Katrina MacLeod, Mark Stopfer, and Michael Wehr \\ Division of Biology \\ California Institute of Technology \\ Pasadena, Califomia 91125 USA
}

A requirement to understand mushroom body (MB) function is to characterize the operations (or transformations) that they impose on incoming signals. Understanding the nature of these integrative operations requires an understanding of the inputs from other brain areas. By inputs we mean not only the anatomical pathways leading to the MBs, but also the dynamic structure of the inflow of sensory (and other) signals. Neurons are complex, capacitative, and generally nonlinear devices that transform barrages of neurochemical packets into electrical waveforms. Their modes of operation are intrinsically time dependent and therefore, their functions or roles in a circuit cannot be inferred only from structural data.

Thanks to elegant anatomical, behavioral, genetic, and molecular (for review, see Crittenden et al. 1998; Hammer and Menzel 1998; Heisenberg 1998; Wolf et al. 1998) studies, there is convincing evidence that MB circuits are involved, at least in fruit flies and honeybees, in some forms of odor integration and learning. In vivo electrophysiological studies of MB neurons, however, are rare and mainly restricted to individual (or small populations of) so-called extrinsic neurons, that is, those whose processes link MBs with other brain areas (Schildberger 1983, 1984; Homberg 1984; Hammer 1993; Mauelshagen 1993; Li and Strausfeld 1997). Kaulen et al. (1984) examined extracellular potentials in the MBs of bees, using current source density analysis, and more recently, Laurent and Naraghi (1994) provided a description of stimulusevoked activity in Kenyon cells (KCs), the intrinsic neurons of the MBs, using intracellular recordings. In this short review we will summarize the recent results from our laboratory in an attempt to provide a description of the spatiotemporal structure of olfactory inputs to the MBs and their intrinsic neurons. We will focus only on the encoding of

${ }^{1}$ Corresponding author. odor quality. We will then speculate on the possible role of MB circuits for olfactory processing.

\section{The Circuits}

The locust olfactory circuits linking antenna to MB are summarized in Figure 1. Olfactory receptor neurons terminate in glomeruli in the ipsilateral antennal lobe $(A L)$ (three glomeruli on average in locusts; Masson and Mustaparta 1990) and contact probably both local (axonless) and projection neurons (LNs and PNs, respectively). In locusts, PNs are multiglomerular and each projects to 10-20 of the 1000 glomeruli found in each AL. In contrast, LNs have diffuse and widespread neurites that occupy most of the AL and appear devoid of conspicuous glomerular dendritic tufts (Laurent 1996b). LNs contact PNs directly and inhibit them at least in part through a picrotoxin (PCT)-sensitive GABA receptor channel (MacLeod and Laurent 1996). PNs project in a tight bundle of 830 axons (Leitch and Laurent 1996) to the ipsilateral lateral protocerebral lobe, sending en-passant collaterals to the MB calyx. These collaterals terminate in a spatially distributed manner (Laurent and Naraghi 1994) so that each PN can contact spiny KCs located in most regions of the calyx (Fig. 1). KCs give off a single small diameter $(\sim 200 \mathrm{~nm})$ axon that bifurcates into the alpha and beta lobes. KCs also contact their immediate neighbors (other KCs) through chemical synaptic contacts along the pedunculus and within each of the two lobes (Schürmann 1974; Leitch and Laurent 1996). They also contact "extrinsic" neurons in the lobes (Schildberger 1984; Li and Strausfeld 1997; Fig. 1).

\section{Global Odor-Evoked Phenomena}

In locusts, delivery of an airborne odor onto an antenna activates a group of PNs and LNs in the

LEARN IN G \& MEM ORY 5:124-132 @ 1998 by Cold Spring Harbor Laboratory Press ISSN 1072-0502/98 \$5.00

$$
\begin{array}{llllllllllllllll}
L & E & A & R & N & I & N & G & \underset{\mathbf{1 2}}{\boldsymbol{X}} & M & E & M & O & R & Y
\end{array}
$$




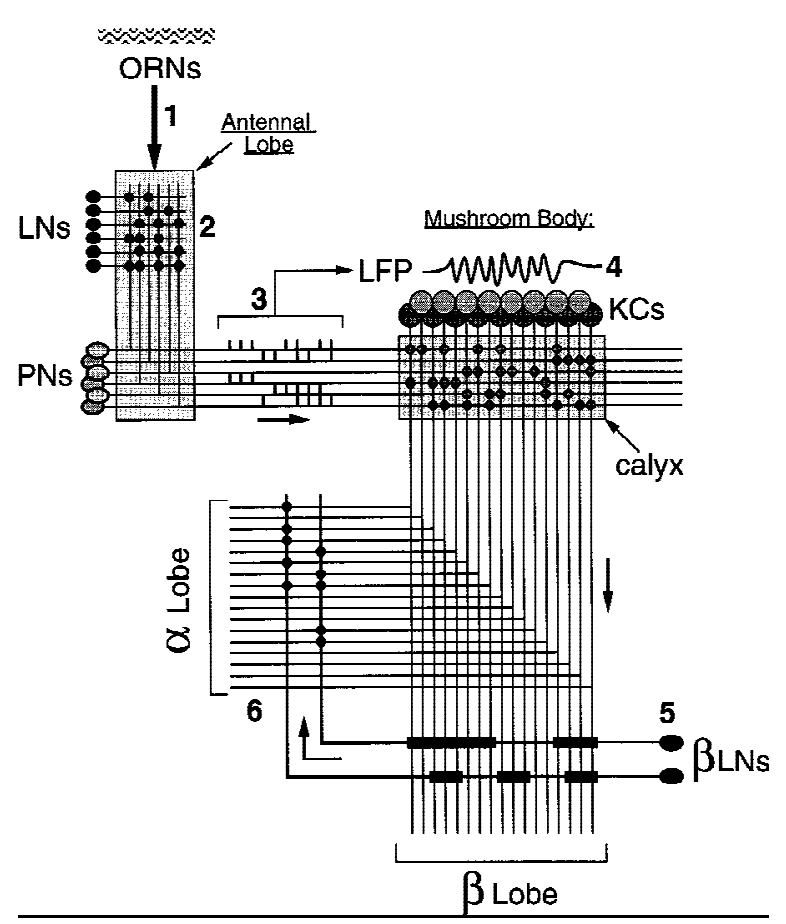

Figure 1: Circuit diagram of the olfactory pathway in the locust brain. O dors are transduced by arrays of olfactory receptor neurons (ORNs) in the antenna (1). ORN s activate ensembles of local and projection neurons (LN s and PNs) in the antennal lobe. LN s inhibit PN s (2) through a fast picrotoxin-sensitive GABAergic synapse. This inhibitory synapse is (at least in part) responsible for the oscillatory synchronization of the PN S activated by an odor. PNs responding to a given odor usually display specific and reliable slow temporal activity patterns (3), superimposed on these oscillatory responses. The patterns are neuron and odor specific. The coactivation of PN s during an odor response causes synchronized and rhythmic EPSPs in KCs, the intrinsic neurons of the mushroom bodies. These synchronized EPSPs can be detected as odor-evoked bouts of 20- to 30$\mathrm{Hz}$ local field potential (LFP) oscillations (4) in the calyx. Arrays of KCs thus activated send action potentials dow $n$ their axons to the alpha and beta lobes. In the beta lobe, the KCs contact beta lobe neurons ( $\beta L N s, 5)$, which send axonal collaterals to the alpha lobe (6).

ipsilateral antennal lobe. The size of the activated PN group was estimated from electrophysiological experiments to be $\sim 10 \%-15 \%$ of the total complement (i.e. $\sim 80-120$ of 830 PNs, number estimated from proportion of sampled PNs that responded in single animals to an odor tested), and appeared not to depend on whether the odor was mono- or multimolecular (Laurent and Davidowitz 1994). Individual PNs can respond and hence, participate in representing different odors, even if these odors do not share molecular compounds. These "spatial" ensembles are thus overlapping and combinatorial (Laurent 1996a,b). If the antennal lobe were a noise-free system, if PNs could be described simply as responding or not responding to an odor, and if downstream decoding networks could differentiate two PN assemblies that differed only by one $\mathrm{PN}$, then the number of combinations (and hence possible odor representations) would be enormous: 830 !/ ( 730 ! $\cdot 100$ !), i.e., $~ 10^{131}$. Neurons and chemical synaptic transmission are intrinsically noisy, however, and therefore, it is unlikely that any of the above three "ifs" apply to brain circuits and neurons. Odor (or pattern) learning, classification, and recognition by the brain are complex problems in part precisely because both signal and processors are noisy.

\section{Temporal Odor-Evoked Activity Patterns}

In addition to the spatial features described above, odor responses of locust antennal lobe PNs display the following temporal characteristics:

\section{OSCILLATIONS}

The responses of PNs to odors contain 20- to 30-Hz membrane potential oscillations composed of fast alternating excitatory postsynaptic potentials (EPSPs) and inhibitory postsynaptic potentials (IPSPs) (Laurent and Davidowitz 1994). Hence, the spikes produced by a PN are often periodic (see below). The IPSPs are caused by GABA-mediated inputs (Christensen et al. 1993; Leitch and Laurent 1996) from connected LNs. LNs oscillate at the same frequency, with a $90^{\circ}$ phase lag relative to the PNs. Blocking the GABAergic synapse betw een LNs and PNs with PCT desynchronizes selectively all antennal lobe neurons (MacLeod and Laurent 1996). Individual PNs receive inputs from many LNs; hyperpolarizing a single $L N$ during an odor response does not significantly alter the oscillatory response of a postsynaptic PN (Laurent and Davidowitz 1994; MacLeod and Laurent 1996).

\section{SYNCHRONIZATION}

The PNs that respond to the same odor fire in (transient, see below) pairwise synchrony. Because their firing is by-and-large periodic, their synchronized spikes provide periodic input to the postsyn- 
aptic $\mathrm{KCs}$ in the MB, producing $20-$ to $30-\mathrm{Hz}$ local field potential (LFP) oscillations in the MB calyx. These oscillations can also be seen as subthreshold $20-$ to $30-\mathrm{Hz}$ oscillations of membrane potential and phase-locked action potentials in intracellularly recorded KCs (Laurent and Naraghi 1994). Odors, but not air alone, evoke such bursts of LFP oscillations (Laurent and Davidowitz 1994). The oscillation frequency, how ever, is independent of the odor.

\section{PHASE}

The phase of PN spikes relative to the corresponding LFP oscillation cycles (or to other responding PNs) varies around a constant mean that is stimulus independent. Spike phase, therefore, contains no information about odor identity (Laurent and Davidowitz 1994; Laurent 1996b; Wehr and Laurent 1996).

\section{PHASE LOCKING}

Not all of the spikes produced by a PN in response to an odor phase lock to the LFP. The spikes that do, how ever, occur in relatively precise and consistent temporal windows during the odor response. The length and timing of these windows differ across neurons for an odor, and across odors for one neuron. Consequently, any two neurons that respond to the same odor may produce phase-locked spikes only during a few cycles of the ensemble response; some PN pairs whose components are both activated by the same odor may never even fire synchronized spikes together (Laurent et al. 1996).

\section{SLOW TEMPORAL PATTERNS}

Slow temporal response patterns are superimposed on these fast odor-evoked PN oscillatory responses. Indeed, individual PNs usually respond to an odor with a specific and characteristic spike profile, composed of several successive periods of activity and silence. Different PNs respond differently to the same odor, and individual PNs respond differently to different odors (Laurent et al. 1996).

\section{PRECISION}

The relative ordering of $\mathrm{PN}$ spikes with respect to the ensemble response in these oscillatory temporal patterns is very precise. Indeed, the response of a PN to two odors A and B may differ only by the rank order of the spikes each odor evokes. For example, they can occur with non-zero probabilities in cycles 1,2,3 of the LFP oscillation for odor A and cycles 2,3,4 for odor B. This indicates that a rate code-that considers average spike numbers over the entire response durationmisses information contained in the relative timing of the spikes within the oscillatory response (Wehr and Laurent 1996).

\section{PN-PN COUPLING}

The firing probability of a PN during a given cycle of its response can be tightly coupled to its firing probability in a different cycle of the same trial. Similarly, the firing probability of a PN during a given cycle is linked to the firing probability of other PNs recorded simultaneously in that or a different cycle of the same trial (Wehr and Laurent 1996). In other words, the firing behaviors of different PNs are not independent during an odor response. The degree to which different PNs are coupled to each other, how ever, varies throughout the response. Therefore, the encoding of odors must be studied in the context of complex circuit dynamics in which PNs probably influence each other's firing behavior.

\section{A Hypothesis for Spatiotemporal Odor Codes in the Antennal Lobe}

A simple mean rate decoding scheme, which assigns significance only to mean spike counts over an extended period and discards spike timing, produces only a fraction of the odor identity information that is contained in the spike trains (Wehr and Laurent 1996). In other words, odor-specific information is contained in precise spatial and temporal aspects of PN firing. Therefore, we propose that space and time (i.e., not only which neurons but also when these neurons are activated) are dimensions of the code for odors. The spatial aspects are contained in the identities of the activated PNs ( $10 \%-15 \%$ of total number of available PNs for most odors tested). An abstract representation of a purely spatial odor code is shown in Figure 2A. The temporal aspects involve two interlocked phenomena: (1) the transient and periodic synchronization of active PNs and (2) the evolution of the odor-coding assemblies along an odor-specific trajectory during an odor response (Fig. 2B). In this

$$
\begin{array}{llllllllllllllll}
L & E & A & R & N & I & N & G & \underset{126}{\mathbf{X}} & M & E & M & O & R & Y
\end{array}
$$



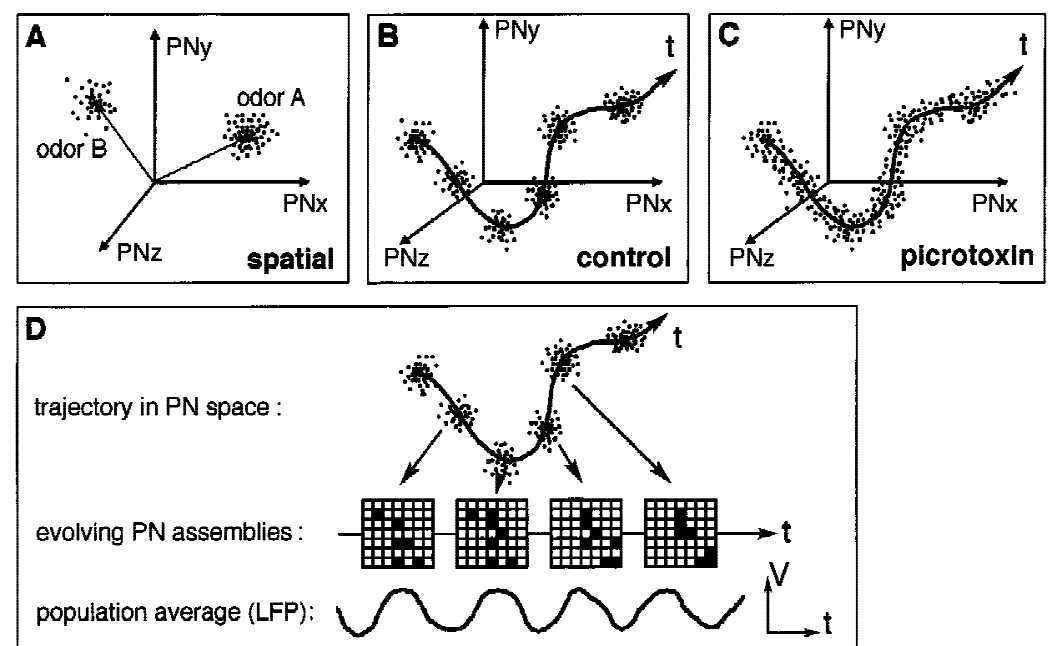

Figure 2: Schematic representations of odor-coding hypotheses in the antennal lobe (the analog of the vertebrate olfactory bulb). $(A-C)$ If we consider the PN s as independent dimensions for the odor code, each odor can be represented within this "PN-space" by clouds of points (spatial code, $A$ ) or trajectories (spatiotemporal code, $B-C$ ). In $A-C$, odor representations have been projected from $\sim 830$ (the number of PNs in the antennal lobe) onto three dimensions (PN $x, y, z)$. In $A$, a spatial code is represented. In this scheme, each odor (e.g. $A$ or $B$ ) can be seen as a vector pointing to a point (or cloud of points, given trial-totrial variability) in PN space. This implies that the activity of each PN is measured

by some average firing rate, or total spike count. In $B$, a spatiotemporal code is represented. In it, each odor is now represented by a trajectory in PN space, defined by a series of vectors (or points). Each one of these vectors corresponds to the state of the network of PNs at each cycle of the oscillation, as explained in D. Each cycle of the LFP oscillation is thus the result of an assembly of synchronized PNs and these PN assemblies evolve throughout the duration of the odor presentation. In $C$, the evolution of the representation in PN space is schematized in a PCT-treated antennal lobe. In this condition, PN s continue to fire with their normal slow temporal patterns (explaining the unchanged overall trajectory), but without the oscillatory synchronization (explaining the absence of clusters corresponding to the cycle-by-cycle assemblies).

hypothesis, the oscillation can be seen as a clock at whose rate the spatial representation is updated during a single odor response (Fig. 2D; Laurent et al. 1996; Laurent 1996b; Wehr and Laurent 1996). For this hypothesis to be true, evidence is needed to prove that both spatial and temporal aspects of the representation are used by the brain for odor perception or learning, or both.

Although the idea that sensory stimuli can be represented combinatorially by distributed ensembles of coarsely tuned neurons is not new (for example, see Salinas and Abbott 1994; Georgopoulos 1995 for representation of hand movement in primate motor cortex or Wilson and McNaughton 1993 for representation of behavioral space in rat hippocampal formation), the hypothesis that spike timing or synchronization plays a role in stimulus encoding has received rather little direct (i.e., establishing a causal, rather than a correlative link) experimental attention (for example, see Abeles 1981; Bialek et al. 1991; O'Keefe and Recce 1993; Gray 1994; Heller et al. 1995; Abbott et al. 1996). Therefore, a causal analysis of this proposed scheme requires, among other things, manipulations of the temporal features of the stimulus representation and observation of the effects of these manipulations on dow nstream circuits and behavior. The ideal experiments for these tests would require at least two manipulations. In the first, the composition of the neural assemblies firing synchronously during each cycle would be left intact, but the order of recruitment of the assemblies would be scrambled. In the second, the oscillatory synchronization of the recruited PNs would be abolished, without change to the mean firing profile of each PN. This second manipulation has recently been accomplished.

\section{Mechanisms for PN Synchronization and Consequences}

The synchronization and phase-locking of locust antennal lobe PNs during odor responses can be blocked selectively by local injection into the antennal lobe of the GABA receptor-gated chloride channel blocker PCT (MacLeod and Laurent 1996). This manipulation blocks the fast IPSPs caused by LNs onto PNs but not the slow and odor-specific temporal patterns seen in PNs during odor responses. The slow, long-lasting inhibition contributing to the odor-specific temporal patterns is unaffected by PCT (MacLeod and Laurent 1996), suggesting that it is mediated by mechanisms independent of this type of GABA receptor-channel. 2-Hydroxysaclophen, an antagonist of verte- 
brate $\mathrm{GABA}_{B}$ receptors, had no effect on the temporal patterns (K. MacLeod and G. Laurent, unpubl.). The mechanisms underlying slow temporal patterns, therefore, likely involve other neurotransmitter-receptor systems, such as histamine (McClintock and Ache 1989), nitric oxide (Müller and Buchner 1993; Gelperin 1994), or even ionic pumps. Conceivably, they could also result from temporal patterning of the antennal afferent input and from complex emergent dynamic behavior of the highly interconnected antennal lobe circuits.

The finding that fast local inhibition is essential for the production of oscillatory synchronization in the AL networks agrees with recent in vitro and computational results showing that inhibitory feedback shapes oscillatory synchronization in mammalian hippocampus (Whittington et al. 1995; Traub et al. 1996a,b; Wang and Buzsáki 1996), thalamus (van Vreeswijk et al. 1995; Bal and McCormick 1996), and cerebral cortex (Lytton and Sejnowski 1991; Connors and Amitai 1997). Our results in locusts, however, had the benefit of being obtained in vivo and in nonanesthetized animals, and showed that the response tuning of PNs was not altered by PCT-mediated desynchronization. It is, to our knowledge, the only preparation thus far in which oscillations in a specific circuit can be abolished in vivo, conditions essential for testing the role of neural synchronization for stimulus learning and discrimination.

\section{Role of Oscillatory Synchronization for Odor Discrimination}

To test for the possible involvement of oscillatory synchronization (and hence of the temporal codes it may carry) for odor discrimination, we used honeybees (Stopfer et al. 1997), which can be trained to extend their mouth parts (probosces) in response to specific odors after a few associative forward pairings of these odors with a sucrose reward [proboscis extension (PE) conditioning] (Kuwabara 1957; Bitterman et al. 1983; Smith and Menzel 1989; Menzel 1990; Hammer and Menzel 1995).

A first series of experiments was designed to determine whether presentation of odors to the antenna of a bee produces the same neural phenomena as those observed in the $A L$ and $M B$ of locusts. These experiments demonstrated that many of the phenomena described above for locusts also apply to bees. Namely, odors evoke syn- chronized $(30 \mathrm{~Hz})$ oscillations of antennal lobe neurons and phase-locked oscillations of field potential in the ipsilateral MB calyx (and alpha lobes); these synchronized oscillations are blocked by PCT (injected into or applied topically onto the antennal lobes), which spares the slow temporal response patterns of PNs (Stopfer et al. 1997). Therefore, PCT can be used in the honeybee to abolish selectively oscillatory synchronization of PNs during odor conditioning (Fig. 2C).

In a second set of experiments, performed in collaboration with Drs. Brian Smith and Seetha Bhagavan (Ohio State University, Columbus), we tested the importance of oscillatory synchronization for odor learning and discrimination, using PCT injection into, or selective application onto, the antennal lobes (Stopfer et al. 1997). We used a PE conditioning assay to test whether PCT could disrupt olfactory discrimination (Bitterman et al. 1983; Smith and Menzel 1989). When forager honeybees experience forward pairing of an odor [conditioned stimulus (CS)] with sucrose reinforcement, their PE response to that specific odor increases dramatically for $48 \mathrm{hr}$ or more. This increase is attributable to associative learning mechanisms (Menzel 1990). In addition, the conditioned response generalizes to some extent to odors that are structurally similar to the CS (Smith and Menzel 1989). For example, once conditioned to an aliphatic alcohol (e.g., 1-hexanol), bees show a somewhat heightened response to other structurally similar alcohols (e.g., 1-octanol). This generalization response is never as strong as the response to the CS itself, but it is greater than the generalization response to structurally dissimilar odorants (e.g., terpenes). We reasoned that if oscillatory synchronization plays a role in odor learning or discrimination, PCT application to the antennal lobe should diminish the ability of bees to discriminate odors and therefore, should increase odor generalization. Animals were divided in two groups: A control (saline-treated) group and a test (PCTtreated) group and the drugs were applied blind (Stopfer et al. 1997). We used recovery intervals of 10, 45, and 60 min between drug treatment and conditioning. Results are described for the 10-min group. Both PCT-and saline-treated groups learned the CS-sucrose pairing equally well (conditioning trials were counterbalanced), showing a maximal response by conditioning trial 5 . Sixty minutes after conditioning, the two groups were given extinction trials with the CS and two additional odors, one similar (S) and the other dissimilar (D)

$$
\begin{array}{llllllllllllllll}
L & E & A & R & N & I & N & G & \underset{128}{\mathbf{X}} & M & E & M & O & R & Y
\end{array}
$$


to the CS. The number of animals in each group that responded with a proboscis extension to CS, $\mathrm{S}$, or D was measured. Saline-treated animals responded significantly less often to $\mathrm{S}$ than they did to the $\mathrm{CS}$, indicating discrimination of the two related odors. In contrast, PCT-treated animals failed to discriminate the CS from $\mathrm{S}$. Like the salinetreated controls, how ever, PCT-treated bees could discriminate the dissimilar odor, geraniol, from the two aliphatic alcohols (CS and S). We concluded that PCT had no significant nonspecific effects on learning but rather that PCT affected more difficult odor discrimination tasks specifically (Stopfer et al. 1997). It appears, therefore, that the temporal structure of the responses of PNs to odors is indeed relevant for odor discrimination and that odor codes contain both spatial and temporal components. We propose that the temporal aspects of the odor codes are used in conditions where the spatial features of the representations overlap greatly (i.e., in cases where the brain must discriminate two stimuli that evoke activity in similar neural assemblies). In such cases, temporal aspects of the recruitment of these neurons would offer additional features by which discrimination could be accomplished. Our results provide the first evidence that neural synchronization is required for the decoding of such messages.

\section{But What of the Decoders?}

That odor-specific temporal response patterns in AL PNs exist and might be required for odor discrimination implies that the brain should be able to decode such temporally coded information. Where and how might such an operation take place? Because PNs send collaterals to the MB calyx, it is possible that KCs play a critical role in processing these olfactory signals. Could these neurons, in some way, play a role as temporal decoders? What would be required of such devices?

Although we have demonstrated that information about odors is contained in the relative timing of PN action potentials and that oscillatory synchronization is required for fine odor discrimination, it remains to be shown directly that specific sequences of PN assemblies are necessary for certain kinds of odor discrimination. It could be, for example, that the brain forms a succession of neural representations-embodied in locusts by the PN assemblies that succeed each other at the rate of one per oscillation cycle (Fig. 2D). Each one of the successive representations would on its own carry (potentially) redundant information about the stimulus. In other words, it could be that the order in which these assemblies succeed each other is not important for downstream decoders, but rather is linked to the stimulus (i.e., stimulus specific) simply because the neural system is somewhat constrained to "visit" these neural states in a specific order. To take a simple metaphor, this model is like considering a string of words with potentially identical meaning but each spoken in a different language. The listener (decoder) would store these words, and later "recognize" the input upon matching one (or more) of these words with a library of stored ones.

On the other hand, it could be that the sequential aspect of the representation is itself important (i.e., usable) and in fact used by an appropriately tuned downstream decoder. To use the language analogy again, it is now as if words in a sentence, or syllables in a word, needed to appear in a specific order to be recognizable. Therefore, could $\mathrm{KCs}$ play a role in this temporal decoding? Let us consider three relevant facts: (1) To be useful for such a function, KCs would need to receive convergent input from many PNs. Our physiological data indicate that this is indeed the case; stepping up electrical stimulation of AL PNs causes incrementally increasing EPSPs in single intracellularly recorded $\mathrm{KCS}$, with at least 10 easily discriminable EPSP amplitudes (Laurent and Naraghi 1994). Anatomical data from Golgi (Kenyon 1896; Mobbs 1982) as well as intracellular stains (Laurent and Naraghi 1994) also suggest that individual KCs must receive vast amounts of synaptic inputs on their spiny calyceal dendrites. (2) The temporal decoder (or circuits/devices upstream) would need to be sensitive to synchrony of its inputs. Indeed, our behavioral results with honeybees indicate that PN synchronization is required for fine odor discrimination (Stopfer et al. 1997). KCs appear to be well suited for such a function. Our in vivo intracellular data from locusts indicate that $\mathrm{KCs}$ behave nonlinearly in response to simultaneous PN inputs. Provided that a minimum number of PNs have been activated synchronously, the resulting KC EPSPs are amplified nonlinearly in a voltage-dependent manner (Laurent and Naraghi 1994). KCs thus appear to be selectively sensitive to simultaneous PN inputs, and by the same token, less sensitive to out-of-phase PN spikes. KCs could, therefore, act as filters, selective for synchronized inputs from AL PNs. (3) KCs would need to be

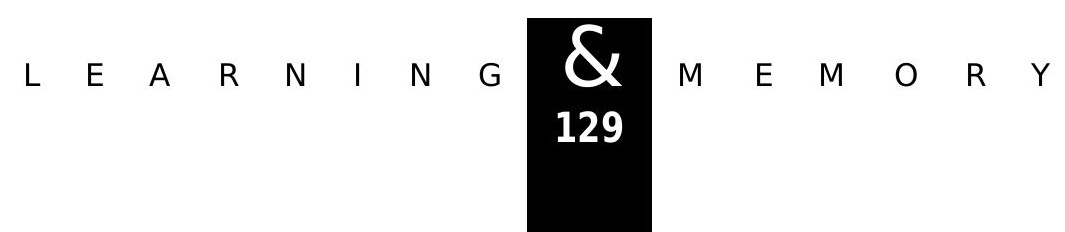


selectively sensitive to sequences of PN inputs. The mechanisms for such temporal selectivity can, at this point, only be imagined (for example, see Buonomano and Merzenich 1995). Let us consider a random $\mathrm{KC}$ that receives inputs from an array of 50 of the 830 PNs (called $\{1,2, \ldots, 50\}$ ) from the antennal lobe. When odor $A$ is presented, a subset of 10 of these 50 PNs is activated in the order $\{1,3,7,49\}\{1,3,7,10,49\}\{3,7,10,12,49\}$. The challenge is to imagine a set of biophysical features that, on their own, would make this cell sensitive to this, rather than a different input sequence. The temporal sensitivity could, in principle, be tuned to sequences of varied lengths. This constitutes a fascinating problem that might be solved not by KCs themselves, but by neurons downstream from them, such as the multimodal extrinsic neurons described in other insects [Schildberger (1984) in crickets; Homberg (1984) in bees; and Li and Strausfeld (1997) in cockroaches]. There is, at this point, no evidence that sequence selectivity exists in either KCs or their immediate targets. A first step will be to assess whether the odor responses (firing pattern and statistics, "tuning" specificity) of KCs and MB extrinsic neurons are sensitive to PN synchronization.

\section{Conclusions}

Our electrophysiological results on odor representation by projection neurons in the locust and honeybee antennal lobes suggest that olfactory inputs to the MBs are structured spatially and temporally in a stimulus-specific manner. A simple way to imagine these spatiotemporal activity patterns is to consider the representation as a dynamic assembly that is updated gradually and rhythmically during an odor presentation (Fig. 2D). Individual KCS, prime targets of PN axonal collaterals in the MB, thus likely receive, over the presentation of an odor, a barrage of inputs from many PNs at any one time and from different PN assemblies at different epochs of the stimulus duration. Behavioral data obtained with honeybees suggest that PN synchronization is not required for odor conditioning in a classical forw ard pairing paradigm. These data suggest, how ever, that PN desynchronization leads to impairments of fine odor discrimination, suggesting that circuits downstream of the antennal lobe must be able to detect the presence or absence of synchronized input for certain (fine) behavioral tasks. Because of the electrical properties of their membrane (assessed by way of current-clamp intracellular recordings in vivo), KCs could act potentially as selective filters, sensitive to the degree of synchronization of the PNs converging onto them. Because not all of the successive spikes produced by individual PNs are phase-locked to the field potential during an odor presentation, KCs could, because of their sensitivity to input coherence, help form a new and sparser representation for each odor in the MB, more suitable for storage, overlap reduction, and later recognition. (Of course, other representations for other purposes may coexist.)

Potentially, the sequential aspects of odorevoked activity patterns could be used for odor coding and decoding,but the mechanisms for such (putative) temporal selectivity in the MBs or elsewhere remain to be discovered. From a different perspective,the dynamic features of the representations could simply be a manifestation of the complex self-organizing behavior of densely interconnected nonlinear elements (the antennal lobe LNs and PNs) (for example, see Freeman 1992; Kelso 1995). It thus appears to us that a better understanding of olfactory coding, memory, and of the function of MBs (as well as that of any equivalent structure in other brains) in these processes, will not be possible without the study of collective neuronal behavior and its emergent properties.

\section{Acknowledgements}

The work described here was made possible by financial support from the National Science Foundation (NSF), the N SF-Presidential Faculty Fellows program, and the Sloan Center for Theoretical Neuroscience at Caltech to G.L. The publication costs of this article were defrayed in part by payment of page charges. This article must therefore be hereby marked "advertisement" in accordance with 18 USC section 1734 solely to indicate this fact.

\section{References}

Abbott, L.F., E.T. Rolls, and M.J. Tovee. 1996.

Representational capacity of face coding in monkeys. Cerebral Cortex 6: 498-505.

Abeles, M. 1981. Corticonics. Neural circuits of the cerebral cortex. Cambridge University Press, Cambridge, UK.

Bal, T. and D.A. McCormick. 1996. What stops synchronized thalamocortical oscillations? Neuron 17: 297-308.

Bialek, W., F. Rieke, R.R. de Ruyter van Steveninck, and D. W arland. 1991. Reading a neural code. Science

252: 1854-1857.

\section{$\begin{array}{llllllllllllllll}L & E & A & R & N & I & N & G & \underset{\mathbf{1 3 0}}{\boldsymbol{X}} & M & E & M & O & R & Y\end{array}$}


Bitterman, M.E., R. M enzel, A. Fietz, and S.J. Schäfer. 1983. Classical-conditioning of proboscis extension in honeybees A pis mellifera. J. Comp. Psychol. 97: 107-119.

Buonomano, D.V. and M.M. Merzenich. 1995. Temporal information transformed into a spatial code by a neural network with realistic properties. Science 267: 1028-1030.

Christensen, T.A., B.R. W aldrop, I.D. Harrow, and J.G. Hildebrand. 1993. Local interneurons and information processing in the olfactory glomeruli of the moth Manduca sexta. J. Comp. Physiol. A 173: 385-399.

Connors, B.W. and Y. Amitai. 1997. Making waves in the neocortex. Neuron 18: 347-349.

Crittenden, J.R., E.M.C. Skoulakis, K.-A. Han, D. Kalderon, and R.L. Davis. 1998. Tripartite mushroom body architecture revealed by antigenic markers. Learn. \& Mem. (this issue).

Freeman, W.J. 1992. Nonlinear dynamics in olfactory information processing. In Olfaction, a model system for computational neuroscience (ed. J.L. Davis and $\mathrm{H}$. Eichenbaum), pp. 225-249. MIT Press, Cambridge, MA.

Gelperin, A. 1994. Nitric oxide mediates network oscillations of olfactory interneurons in a terrestrial mollusc. Nature 369: 61-63.

Georgopoulos, A.P. 1995. Current issues in directional motor control. Trends Neurosci. 18: 506-510.

Gray, C.M. 1994. Synchronous oscillations in neuronal systems: Mechanisms and functions. J. Comput. Neurosci. 1: 11-38.

Hammer, M. 1993. An identified neuron mediates the unconditioned stimulus in associative olfactory learning in honeybees. Nature 366: 59-63.

Hammer, M. and R. Menzel. 1995. Learning and memory in the honey-bee. J. Neurosci. 15: 1617-1630.

- - . 1998. Multiple sites of associative odor learning as revealed by local brain microinjections of octopamine in honeybees. Learn. \& Mem. (this issue).

Heisenberg, M. 1998. What do the mushroom bodies do for the insect brain? An introduction. Learn. \& Mem. (this issue).

Heller, J., J.A. Hert, T.W. Kjaer, and B.J. Richmond. 1995. Information flow and temporal coding in primate pattern vision. J. Comput. Neurosci. 2: 175-193.

Homberg, U. 1984. Processing of antennal information in extrinsic mushroom body neurons of the bee brain. J. Comp. Physiol. A 154: 825-836.

Kaulen, P., J. Erber, and P. Mobbs. 1984. Current source-density analysis in the mushroom bodies of the honeybee Apis mellifera. J. Comp. Physiol. A 154: 569-582.
Kelso, J.A.S. 1995. Dynamic patterns. The self organization of brain and behavior. MIT Press, Cambridge, MA.

Kenyon, F.C. 1896. The brain of the bee. A preliminary contribution to the morphology of the nervous system of the Arthropoda. J. Comp. Neurol. 6: 133-210.

Kuwabara, M. 1957. Bildung des bedingten Reflexes von Pavlovs Typus bei der Honigbiene, Apis mellifica. J. Fac. Sci. Hokkaido Univ. (Ser. VI Zool.) 13: 458-464.

Laurent, G. 1996a. O dor images and tunes. Neuron 16: $473-476$.

- - . 1996b. Dynamical representation of odors by oscillating and evolving neural assemblies. Trends Neurosci. 19: $489-496$.

Laurent, G. and H. Davidowitz. 1994. Encoding of olfactory information with oscillating neural assemblies. Science 265: 1872-1875.

Laurent, G. and M. Naraghi. 1994. O dorant-induced oscillations in the mushroom bodies of the locust. J. Neurosci. 14: 2993-3004.

Laurent, G., M. Wehr, and H. Davidowitz. 1996. Temporal representation of odors in an olfactory network. J. Neurosci. 16: 3837-3847.

Leitch, B. and G. Laurent. 1996. GABAergic synapses in the antennal lobe and mushroom body of the locust olfactory system. J. Comp. Neurol. 372: 487-514.

Li, Y.S. and N.J. Strausfeld. 1997. Morphology and sensory modality of mushroom body extrinsic neurons in the brain of the cockroach Periplaneta americana. J. Comp.Neurol. 387: $631-650$.

Lytton, W.W. and T.J. Sejnowski. 1991. Simulations of cortical pyramidal neurons synchronized by inhibitory interneurons. J. Neurophysiol. 66: 1059-1079.

MacLeod, K. and G. Laurent. 1996. Distinct mechanisms for synchronization and temporal patterning of odor-encoding neural assemblies. Science 274: 976-979.

Masson, C. and H. Mustaparta. 1990. Chemical information processing in the olfactory system of insects. Physiol. Rev. 70: 199-245.

Mauelshagen, J. 1993. N eural correlates of olfactory learning-paradigms in an identified neuron in the honeybee brain. J. Neurophysiol. 69: 609-625.

McClintock, T.S. and B.W. Ache. 1989. Histamine directly gates a chloride channel in lobster olfactory receptor neurons. Proc. Natl. Acad. Sci. 86: 8137-8141.

Menzel, R. 1990. Learning, memory and "cognition" in honey bees. In Neurobiology of comparative cognition (ed. R.P. Kesner and D.S. OIton), pp. 237-292. Erlbaum, Hillsdale, NJ.

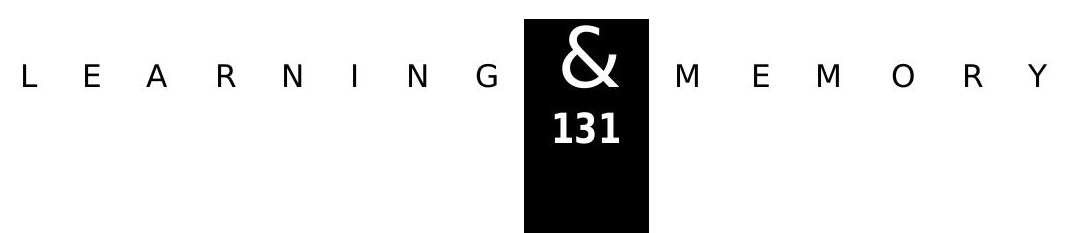




\section{Laurent et al.}

Mobbs, P.G. 1982. The brain of the honeybee Apis mellifera. I. The connections and spatial organization of the mushroom bodies. Philos. Trans. R. Soc. Lond. (Biol.) 298: 309-354.

Müller, U. and E. Buchner. 1993. Histochemical localization of NADPH-diaphorase in the adult Drosophila brain: Is nitric oxide a neuronal messenger also in insects? Naturwissen 80: $524-526$.

O'Keefe, J. and M. Recce. 1993. Phase relationship between hippocampal place units and the EEG theta-rhythm.

Hippocampus 3: 317-330.

Salinas, E. and L.F. Abbott. 1994. Vector reconstruction from firing rates. J. Comput. Neurosci. 1: 89-107.

Schildberger, K. 1983. Local interneurons associated with the mushroom bodies and the central body in the brain of Acheta domesticus. Cell Tissue Res. 230: 573-586.

- - - 1984. Multimodal interneurons in the cricket brain: Properties of identifed extrinsic mushroom body cells. J. Comp. Physiol. A 154: 71-79.

Schürmann, F.-W. 1974. Bemerkungen zur Funktion der Corpora pedunculata im Gehirn der Insekten aus morphologischer Sicht. Exp. Brain Res. 19: 406-432.

Smith, B.H. and R. Menzel. 1989. The use of electromyogram recordings to quantify odorant discrimination in the honey bee, Apis mellifera. Ethology 82: 68-81.

Stopfer, M., S. Bhagavan, B. Smith, and G. Laurent. 1997. Impaired odor discrimination on desynchronization of odor-encoding neural assemblies. Nature 390: 70-74.

Traub, R.D., M.A. Whittington, I.M. Stanford, and J.G.R. Jefferys. 1996a. A mechanism for generation of long-range synchronous fast oscillations in the cortex. Nature 383: $621-624$.

Traub, R.D., M.A. Whittington, S.B. Colling, G. Buzsáki, and J.G.R. Jefferys. 1996b. Analysis of gamma rhythms in the rat hippocampus in vitro and in vivo. J. Physiol. 493: 471-484.

van Vreeswijk, C., L.F. Abbott, and G.B. Ermentrout. 1995. When inhibition, not excitation synchronizes neural firing. $J$. Comput. Neurosci. 1: 313-322.

Wang, X.-J. and G. Buzsáki. 1996. Gamma oscillation by synaptic inhibition in a hippocampal interneuronal network model. J. Neurosci. 16: 6402-6413.

Wehr, M. and G. Laurent. 1996. O dour encoding by temporal sequences of firing in oscillating neural assemblies. Nature 384: 162-166.

Whittington, M.A., R.D. Traub, and J.G.R. Jeffreys. 1995. Synchronized oscillations in interneuron networks driven by metabotropic glutamate receptor activation. Nature 373: 612-615.

Wilson, M.A. and B.L. MCNaughton. 1993. Dynamics of the hippocampal ensemble code for space. Science 261: 1055-1058.

Wolf, R., T. Wittig, L. Liu, G. Wustmann, D. Eyding, and M. Heisenberg. 1998. Drosophila mushroom bodies are dispensable for visual, tactile, and motor learning. Learn. \& Mem. (this issue).

Received January 22, 1998; accepted in revised form April 14, 1998. 


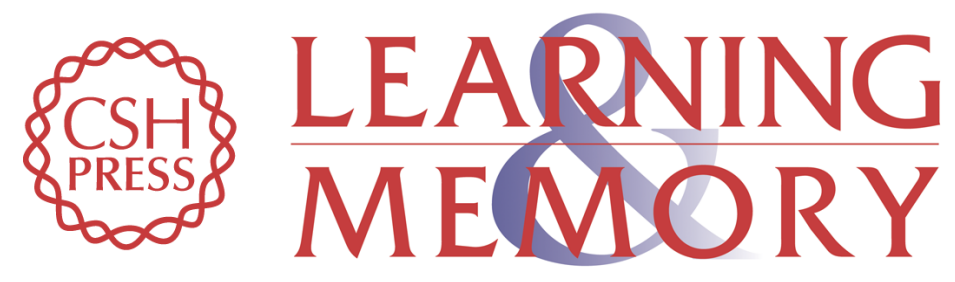

\section{Spatiotemporal Structure of Olfactory Inputs to the Mushroom Bodies}

Gilles Laurent, Katrina MacLeod, Mark Stopfer, et al.

Learn. Mem. 1998, 5:

Access the most recent version at doi:10.1101//m.5.1.124

References This article cites 44 articles, 10 of which can be accessed free at: http://learnmem.cshlp.org/content/5/1/124.full.html\#ref-list-1

License

Email Alerting Receive free email alerts when new articles cite this article - sign up in the box at the Service top right corner of the article or click here. 